\title{
Human Plantar Pressure Image and Foot Shape Matching
}

\author{
A. K. Chong1, J. A. Al-Baghdadi'2, P. Milburn1 \\ ${ }^{1}$ University of Southern Queensland, Toowoomba, Queensland, Australia \\ ${ }^{2}$ Technical College-Baghdad, Baghdad, Iraq \\ Email: chonga@usq.edu.au
}

Received April 2015

\begin{abstract}
Medical doctors and podiatrists have been using plantar pressure for the evaluation of the foot loading in regard to foot biomechanics and foot health for decades. High plantar pressure can cause pain and foot injury such as plantar ulcer during walking. Two types of systems are suitable for pressure capture and they are commonly known as floor mat or floor pad and in-shoe pad. For the floor-mat system pressure values are displayed as image which does not depict the foot shape. Therefore, shortcoming of this system for plantar pressure recording during walking is that the location of the pressure value related to the plantar surface is only approximate. A research was conducted to determine whether markers can be placed on the plantar surface and their positions identifiable on the pressure image. This paper describes the technique for matching the plantar shape to the pressure image during walking.
\end{abstract}

\section{Keywords}

Foot, Pressure, Shape, Image

\section{Introduction}

\subsection{Background}

The Plantar pressure measures (centre of pressure, pressure gradient, impulse, contact duration, pressure-time integral, and centroid of area of contact trajectory) can be obtained by a variety of systems. These systems are electronic devices designed to measure the pressure caused by interaction between the foot and the support surface ((Sih, 2001; Perttunen, 2002; Noce, 2005; Bryant et al., 2005; Caudert et al., 2006; Martínez-Nova et al., 2008; Flórez \& Velásquez, 2010; Al-Baghdadi et al., 2011; Abdul-Razak et al., 2012) [1]-[9]. Normally, the spatial and temporal resolutions of the data recorded by commercial pedobarographic (plantar pressure) systems range from approximately 3 to $10 \mathrm{~mm}$ and 25 to $500 \mathrm{~Hz}$, respectively. The higher resolution systems consist of larger number of small pressure sensors or pressure sensel per unit area. These sensors (Figure 1(a)) are ideal for measuring plantar pressure without disturbing patient movement during trial because they are thin enough to enable non-intrusive measurement (Noce, 2005; Bryant et al., 2005; Abdul-Razak et al., 2012; Rodrigo et al., 2013) [3] [4] [9] [10]. These system are utilised in clinical gait analysis (Abdul-Razak et al., 2012; Bryant et al., 


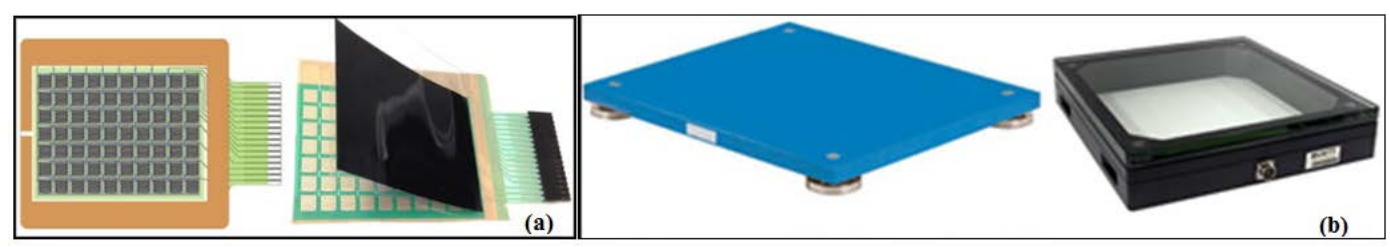

Figure 1. Category of plantar pressure capture system. (a) The matrix of force resistance sensors (FRS) make-up of a pressure-based system; (b) Force-plate system.

2005; Rana, 2009) [4] [9] [11], foot ailment diagnoses and rehabilitation (Abdul-Razak et al., 2012) [9], footwear design, biomechanics analysis (Rana, 2009) [11] and a large number of sport and exercise applications (Orlin \& McPoil, 2000) [12]. A number of scientific studies have reported that these systems can give sufficient results in analyzing human gait compared to the more accurate data of the force plate system (Figure 1(b)) (Noce, 2005) [3]. However, the lack of accuracy (ranges approximately between $\pm 5 \%$ to $\pm 25 \%$ ), uncertainty and variations in sensors' readings, especially during the dynamic measurements, are the major limitations of pressure measurement systems (Orlin \& McPoil, 2000; Abdul-Razak et al., 2012) [9] [12].

In general, two types of pressure recording systems are available for human foot study, namely: floor mat and in-shoes pad. The advantages and the drawbacks of these are detailed below.

\subsection{Floor-Mat Pressure Recording System}

They are utilized for measuring static or dynamic pressure and vertical force applied to plantar surface (Orlin \& McPoil, 2000; Abdul-Razak et al., 2012) [9] [12]. These systems are designed in various shape, dimensions, and resolutions to accommodate a broad range of applications. Nonetheless, the systems consist of many small electronic pressure sensors arranged in a matrix form on a circuit layer which is commonly inserted between two heat stable polyester layers (Figure 1(a)). Using floor-mat systems for gait studies has a number of advantages. The system is suitable for capturing barefoot movement because the pressure mat is thin enough to enable nonintrusive measurement and is ideal for measuring forces without disturbing the dynamics of test subjects (Orlin \& McPoil, 2000; Abdul-Razak et al., 2012) [9] [12]. In addition, the high density of pressure sensors in a mat system allows for precise capturing and transmitting of pressure information (Orlin \& McPoil, 2000) [12].

Normally, this system can record the vertical force as accurately as force plate recording (Figure 2(a)) because the mat system is usually placed on a flat surface parallel to the supporting ground surface similar to the force-plate system (Orlin \& McPoil, 2000) [12]. These systems are more suitable for recording the pressure of bare feet in the indoor environments but they have limitations in the analysis of long period activities such daily exercise and they require a large space with a proper setup because the mat needs to be placed on a walkway to ensure that the tested subject achieve a steady-state gait (minimum three steps are taken) before reaching the mat (Sih, 2001) [1]. The other drawback is the limited accuracy in correlating the foot profile (Cavanagh \& Rodgers, 1987) [13] with the plantar pressure image because there is no distinguishable marker for registering the plantar surface and the image.

\subsection{In-Shoe System}

In-shoe pressure-based systems (Figure 2(b)) are exploited to record pressure between human sole surface and in-sole of footwear (Orlin \& McPoil, 2000; Abdul-Razak et al., 2012; Muro-de-la-Herran et al., 2014) [9] [12] [14]. These systems are indispensable for many medical and sports applications which involves footwear such as shoe design and orthotic design (Abdul-Razak et al., 2012; Muro-de-la-Herran et al., 2014) [9] [14] and sports and exercises (Orlin \& McPoil, 2000) [12]. They consist of pressure film placed inside the shoe (Figure 2) connected to a storage device via USB cable or Bluetooth transmitter. A pressure film is usually a thin sheet which contains a large number of small pressure sensors or sensels (Figure 1). Sensor distribution in the pressure film is dense (high resolution) in the areas of the forefoot and rearfoot region, and tends to have less density (low resolution) in the midfoot area. The system design is flexible, thus a number of manufacturers introduced different fabrication models of in-shoe systems (Noce, 2005; Bamberg et al., 2008; Rana, 2009) [3] [11] [15].

In-shoe pressure-based systems for measuring foot plantar pressure have a number of benefits and drawbacks. They are suitable for recording pressure and interaction acting between the foot and shoe. These systems are 


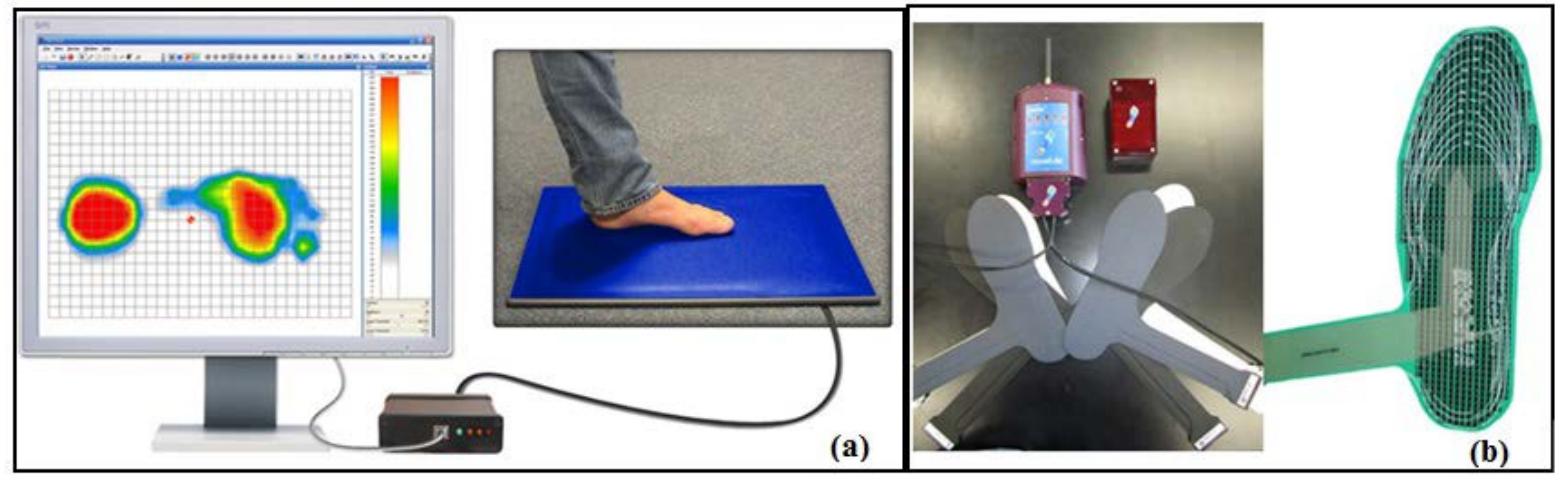

Figure 2. Type of plantar pressure capture system. (a) Floor-mat; (b) In-shoe system.

contained inside the participant's shoes, thus allowing data collection in-doors and out-doors. Additionally, inshoe systems are typically less expensive than floor-based system as smaller area is needed to be covered inside shoes. However, in-shoe systems have lower resolution and are more likely to have a mechanical failure because the connecting cables linking the sensors to each other (or to the cables) can be bent or stretched during foot movement (Orlin \& McPoil, 2000; Muro-de-la-Herran et al., 2014) [12] [14]. In-shoe systems tend to give low accuracy outputs compared with mat systems because the sensor in the in-shoe film is aligned on a non-flat surface inside footwear (Orlin \& McPoil, 2000) [12]. Furthermore, sensor characteristics and sensor performance can be affected and damaged by the humidity and hot environment inside the footwear (Abdul-Razak et al., 2012; Muro-de-la-Herran et al., 2014) [9] [14].

\subsection{Matching the Foot Shape to the Pressure Image}

In this investigation, only floor-mat system was tested because in-shoe system is easier to match. The in-shoe sensor pad is confined within the shoe, thus ink-based marker can be applied efficiently. This paper discusses the methods developed and the results of the data analysis.

\section{Equipment}

\subsection{Tekscan MatScan Floor Based Pressure Mat}

The Tekscan MatScan device utilizes matrix-based pressure sensing systems and single load cell configurations. It is a low profile floor mat (5 mm thick) consisting of 8,448 individual pressure sensing locations, which are referred to as "sensing elements", "sensels", or "cells." with a sampling frequency of $100 \mathrm{~Hz}$. In addition to the plantar pressure, MatScan captures the sway parameters as centre of force (COF) excursions in an antero-posterior (AP) and medio-lateral (ML) direction. These parameters are identified as area, direction of sway, distance and direction travelled by the COF and variability of distance travelled by the COF. The device uses a wireless technology for running it and downloading the captured data.

\subsection{Gait Platform}

A $4.5 \mathrm{~m}$ long gait platform was fabricated to provide proper surface for the trial. The platform allows the tilting of the pressure mat up to 1:50 slope in all four cardinal directions (front, back, left side and right side).

\section{Methods}

\subsection{Pre-Trial Preparation}

The use of human subject in this investigation was approved by the University Ethic Committee (H14REA156). The participants’ right foot (three healthy adult males, ages $37 \pm 3.5$ years, heights $165 \pm 6.5 \mathrm{~cm}$, and body masses $72 \pm 7 \mathrm{~kg}$ ) were marked with foot axis and pressure targets were attached to four locations (end of heel, heel-end of the longitudinal arch, forefoot-end of the longitudinal arch and forefoot at the $2^{\text {nd }}$ metatarsal joint) along the foot central profile line. 


\subsection{Normal Gait Trial}

In the trial, the participants were shown the three-step normal gait across the floor-mat. Before the recording the trial, each participant was given 5 minutes to practise the procedure. In the first stage, no pressure-mark was attached to the plantar surface. The participant walked on the stage with the right foot stepping on the floor mat. In the second stage, pressure-mark was attached to the plantar surface. Again, the participants performed the three-step gait with the right foot stepping on the floor-mat.

\subsection{Correlating the Plantar Pressure Image with the Foot Profile}

The recorded pressure movies (image) were analysed in F-Scan Research ver.6-70-03 software. Using the plantar pressure 2D contour image as the background and the centre of pressure (COP), the foot profile line was inserted digitally. The profile line was established by calculating the coordinates of the pressure-marker locations and inserting these coordinates in the image. The foot profile was established by connecting these markers using a coloured line.

\section{Results and Analysis}

Figure 3 shows the plantar pressure images of (a) no-target gait and (b) targeted gait of the participants. In Subject A's image (b) the COP trajectory and the marked foot axis line were alongside each other. For subjects B and $\mathrm{C}$, the COP and the foot axis lines were quite far apart. Thus, the figures show that the COP trace can be at a different location to the foot axis line. Having this reference line in all the images may provide information on the change of COP location of the foot in subsequent patient's plantar pressure evaluation. Table 1 shows the statistical results of 15 sets observations for the trials involving: 1) no marker; and 2) marker attached taken over two days. The headings in the Table represent minimum pressure, maximum pressure, impulse, peak pressure and mean pressure. These measures were extracted from the images using F-Scan Research ver. 6-70-03 software. The statistics between the non-targeted and targeted samples show insignificant differences in the means and the variances for all the captured data.

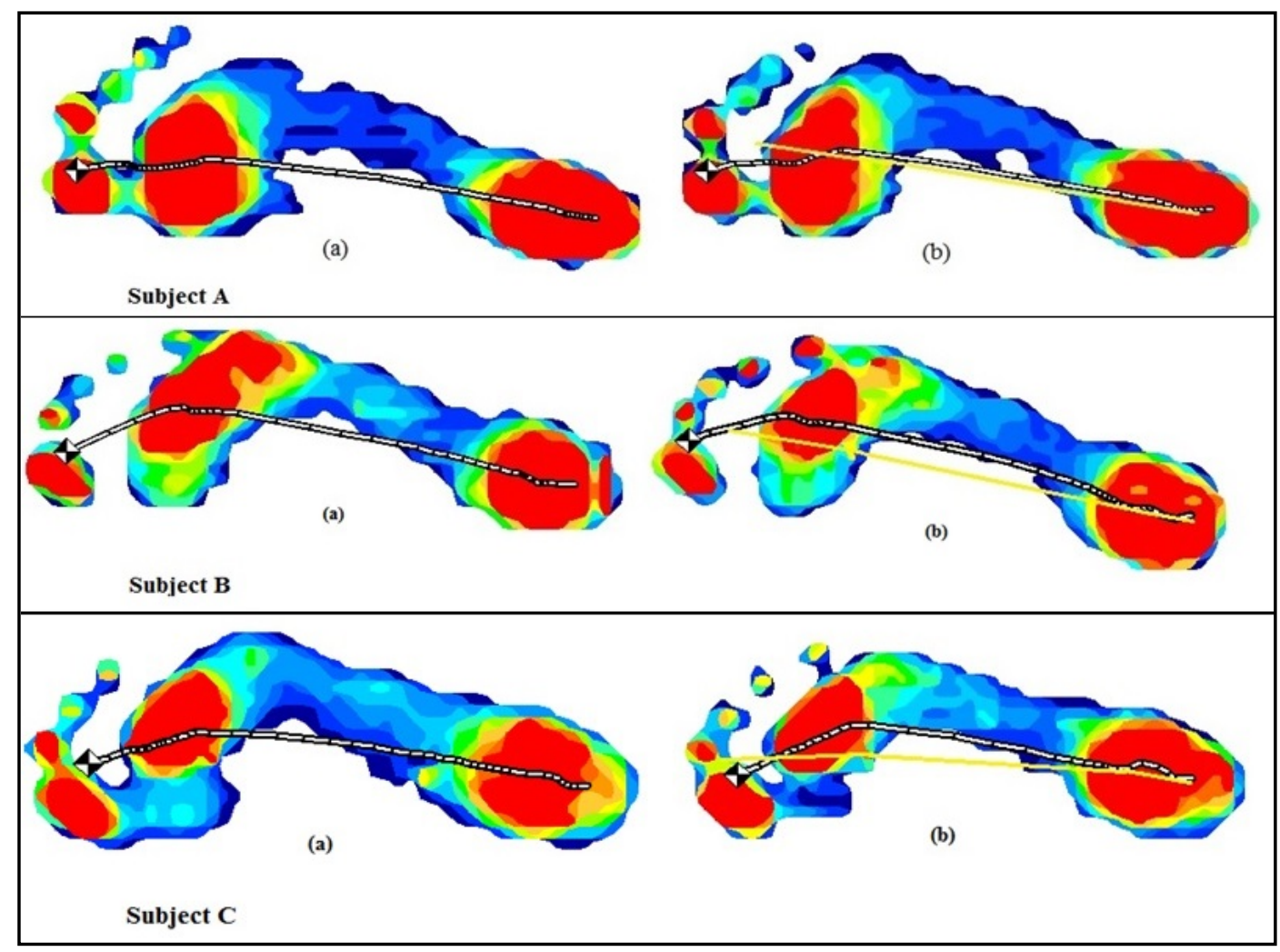

Figure 3. Foot axis and COP trace. (a) No pressure marker; (b) Pressure marker attached. 
Table 1. Statistical tests: Non-targeted sample (1) and targeted sample (2).

\begin{tabular}{cccccc}
\hline Type of pressure data & Min & Max & Impulse & Peak & Mean \\
\hline F-test: variance comparison & $\sigma_{1}^{2}=\sigma_{2}^{2}$ & $\sigma_{1}^{2}=\sigma_{2}^{2}$ & $\sigma_{1}^{2}=\sigma_{2}^{2}$ & $\sigma_{1}^{2}=\sigma_{2}^{2}$ & $\sigma_{1}^{2}=\sigma_{2}^{2}$ \\
t-test: mean comparison & $\mu_{1}-\mu_{2}=0$ & $\mu_{1}-\mu_{2}=0$ & $\mu_{1}-\mu_{2}=0$ & $\mu_{1}-\mu_{2}=0$ & $\mu_{1}-\mu_{2}=0$ \\
\hline
\end{tabular}

\section{Discussion}

There have been no studies to indicate the development of a suitable technique to correlate the plantar pressure image with the foot profile line during normal gait. In a preliminary investigation using ten participants, the plantar pressure image did not resemble the plantar shape in $40 \%$ or 4 of the participants studied. Also, the position of the COP trace of the 2D pressure image varies in location with reference to the edge of the foot in the image between trials. The inability to accurately correlating the foot profile with the pressure image can produce sizeable error in relation to the determination of change of peak pressure position or COP trace for re-occurring plantar pressure evaluation. The statistics of the non-targeted and targeted samples show insignificant differences in the means and variances for a number of pressure data such as the minimum pressure, the maximum pressure, the mean pressure, impulse and peak pressure. Therefore, the use of pressure markers to determine the foot profile in the first instant before other trials of pressure evaluation, can be very useful.

\section{Conclusions}

The research aims to develop a method for correlating the plantar surface with the plantar pressure image captured by floor-based pressure mat. The purpose of correlating the data was to determine whether new useful data can be obtained for the study of change in gait dynamics. Four small-round pressure-targets were placed on the foot profile line of three participants and plantar pressure images were recorded for 15 sets of gaits. The study shows that the method developed is accurate and efficient in correlating the pressure image and the foot shape during walking.

As an extension of this research, the investigation will involve the determination of the effect for a larger group of participants. Also, we will evaluate these new data for foot biomechanics and foot health applications.

\section{References}

[1] Sih, B.L. (2001) Improving Accuracy of the F-Scan Sensor. Jaycor Report, San Diego, CA, 3150.32-01-143.

[2] Perttunen, J. (2002) Foot Loading in Normal and Pathological Walking. University of Jyväskylä.

[3] Noce, H.P. (2005) An In-Shoe Instrument for Acquisition and Storage of Plantar Pressure. MSc Thesis, Oregon State University, USA.

[4] Bryant, J., Stevenson, J., Pelot, R, Morin, E. and Deakin, J. (2005) Development of a Dynamic Biomechanical Model for Load Carriage: Phase 4, Part C3: Dynamic Assessment of Pressure Measurement Systems for Use in Human Load Carriage, Queen’s Univ Kingston (Ontario). Ergonomics Research Group, Toronto, 1-12.

[5] Coudert, P., Vacher, P., Smits, C. and Van Der Zande, M. (2006) A Method to Obtain 3D Foot Shape Deformation during the Gait Cycle. Ninth International Symposium on the 3D Analyses of Human Movement, Valenciennes, Frances.

[6] Martinez-Nova, A, Huerta, J.P. and Sanchez-Rodriguez, R. (2008) Cadence, Age, and Weight as Determinants of Forefoot Plantar Pressures Using the Biofoot In-Shoe System. Journal of the American Podiatric Medical Association, 98, 302-310. http://dx.doi.org/10.7547/0980302

[7] Flórez, J. and Velasquez, A. (2010) Calibration of Force Sensing Resistors (FSR) for Static and Dynamic Applications. IEEE ANDESCON Conference, Bogota, 15-17 September 2010, 1-6. http://dx.doi.org/10.1109/andescon.2010.5633120

[8] Al-Baghdadi, J.A., Chong, A.K., McDougall, K., Alshadli, D., Milburn, P. and Newsham-West, R. (2011) A Dense Surface Modelling Technique for Foot Surface Imaging, Proceedings of the Surveying \& Spatial Sciences Biennial Conference, 21-25 November 2011, 295-302.

[9] Abdul Razak, A.H., Zayegh, A., Begg, R.K. and Wahab, Y. (2012) Foot Plantar Pressure Measurement System: A Review. Sensors, 12, 9884-9912. http://dx.doi.org/10.3390/s120709884

[10] Rodrigo, A.S., Goonetilleke, R.S. and Xiong, S. (2013) Load Distribution to Minimise Pressure-Related Pain on Foot: A Model. Ergonomics, 56, 1180-1193. http://dx.doi.org/10.1080/00140139.2013.797111

[11] Rana, N. (2009) Application of Force Sensing Resistor (FSR) in Design of Pressure Scanning System for Plantar Pres- 
sure Measurement. Proceeding of the 2nd International Conference on Computer and Electrical Engineering, Dubai, 28-30 December 2009, 678-685.

[12] Orlin, M.N. and McPoil, T.G. (2000) Plantar Pressure Assessment. Physical Therapy, 80, 399-409.

[13] Cavanagh, P.R. and Rodgers, M.M. (1987) The Arch Index: A Useful Measure from Footprints. J Biomech, 20, 547551. http://dx.doi.org/10.1016/0021-9290(87)90255-7

[14] Muro-de-la-Herran, A., Garcia-Zapirain, B. and Mendez-Zorrilla, A. (2014) Gait Analysis Methods: An Overview of Wearable and Non-Wearable Systems, Highlighting Clinical Applications. Sensors, 14, 3362-3394. http://dx.doi.org/10.3390/s140203362

[15] Bamberg, S.J.M., Benbasat, A.Y., Scarborough, D.M., Krebs, D.E. and Paradiso, J.A. (2008) Gait Analysis Using a Shoe-Integrated Wireless Sensor System, Information Technology in Biomedicine. IEEE Transactions, 12, 413-423. 